\title{
MODELO DE IMPLEMENTACIÓN DE SOLUCIONES DE COMERCIO ELECTRÓNICO
}

\author{
Giovanny Tarazona Bermúdez* \\ Víctor Hugo Medina García** \\ Lylliana Giraldo ${ }^{* * *}$
}

Recibido: 25/01/2013

Aprobado: 25/10/2013

\begin{abstract}
RESUMEN
El auge del comercio electrónico en la industria mundial y su impacto en la estructura organizacional, han llevado al desarrollo de múltiples soluciones informáticas de manera paralela al comercio tradicional. La incursión en comercio electrónico requiere evaluar condiciones iniciales en la empresa para lograr su éxito. Con el objetivo de disminuir el fracaso que implican inversiones no planificadas en tecnología, se presenta en este artículo de reflexión, un modelo de evaluación de aspectos críticos al momento de implementar una solución de comercio electrónico. La propuesta tiene un enfoque integral sustentado en instrumentos cualitativos y cuantitativos útiles en el diagnóstico.
\end{abstract}

Palabras clave: comercio electrónico, estrategia de negocio, analítica web, ERP, Web 2.0

Doctor en Servicios Informáticos para Internet de la Universidad de Oviedo, DEA en Informática de la Universidad Pontificia de Salamanca, especialización en Ingeniería de Software de la Universidad Distrital. Ingeniero Industrial de la Universidad Distrital. Docente de la Especialización en Ingeniería de Software e Informática Industrial y del programa de Ingeniería Industrial en la Universidad Distrital "Francisco José de Caldas”. Carrera 7 No 40-53 Piso 5, Teléfono 3017907883, Bogotá. gtarazona@udistrital.edu.co

** Doctor en Informática de la Universidad Pontificia de Salamanca. Maestría en Informática de la Universidad Politécnica de Madrid. Especialización en Marketing de la Universidad del Rosario. Ingeniero de Sistemas de la Universidad Distrital. Docente del Doctorado en Ingeniería, Maestría en Ciencias de la Información y las Comunicación y del programa de Ingeniería de Sistemas de la Universidad Distrital “Francisco José de Caldas". Carrera 7 No 40-53 Piso 4, Teléfono 3133338842, Bogotá. vmedina@udistrital.edu.co

*** DEA en el Doctorado en Informática de la Universidad Pontificia de Salamanca. Ingeniera de Sistemas de la Universidad de Medellín. Docente del programa de Ingeniería de Sistemas en la Universidad de Medellín. Teléfono 3147288152.1mgiraldo@udem.edu.co 


\title{
IMPLEMENTATION MODELS OF ELECTRONIC COMMERCE SOLUTIONS
}

\begin{abstract}
The boom of electronic commerce in the global industry and its impact on the organizational structure has led to the development of several information technology solutions in parallel with traditional commerce. Involvement in electronic commerce requires the evaluation of initial conditions in a company in order to succeed. With the purpose of reducing failures which imply unplanned investments in technology, this reflection article shows a model to evaluate critical aspects at the time of implementing an electronic commerce solution. The proposal has an integral approach supported by useful diagnostic qualitative and quantitative instruments.
\end{abstract}

Key words: Electronic commerce; business strategy; web analytics; ERP; Web 2.0. 


\section{INTRODUCCIÓN}

Las empresas se ven obligadas a implementar cambios en su organización para aprovechar al máximo las oportunidades que les brinda el comercio electrónico; de lo contrario, se verán forzados a la quiebra. Por lo tanto, la aplicación efectiva de la estrategia de transformación de negocios electrónicos resulta ser un factor crítico para obtener una ventaja competitiva sostenible [1].

Los sistemas de información y la estrategia de tecnología de información (TI) suelen depender de una estrategia de negocio. Una aplicación tecnológica por sí sola no es la solución, si no obedece a una realidad empresarial; las posibilidades de éxito serán proporcionales a la planeación de la solución de manera coherente con la planeación corporativa; la alineación de las dos estrategias es clave para la mejora de la función de planeación. Desde una perspectiva externa de negocios competitivos, la alineación de TI es el grado en que la estrategia de TI permite e impulsa la estrategia de negocio [2]. La implementación de una solución de comercio electrónico debe obedecer a un ejercicio de planificación, en el cual es necesario identificar el grado de preparación empresarial [3].

Por lo tanto, se propone un modelo de organización para la planeación estratégica del e-Commerce denominado: Modelo PESCE, el cual facilita un método para permitir a las empresas evaluar sus condiciones organizacionales y técnicas para implementar una solución de comercio electrónico coherente con su plan de negocios.

\section{FUNDAMENTACIÓN DEL COMERCIO ELECTRÓNICO}

El comercio electrónico es la compra y venta de productos o servicios a través de medios electrónicos, tales como Internet y otras redes informáticas; su implementación ofrece ventajas a vendedores y compradores; la aplicación y uso de comercio electrónico facilita a vendedores acceder a segmentos de mercados estrechos que se distribuyen ampliamente, mientras que los compradores pueden beneficiarse al acceder a los mercados mundiales con mayor disponibilidad de los productos de una variedad de ofertas a costos reducidos. Esta situación mejora la calidad del producto y la creación de nuevos formas de negocio [4]. Originalmente, el término se aplica a la ejecución de operaciones a través de las transacciones electrónicas, tales como intercambio electrónico de datos. Sin embargo, con el advenimiento de Internet a mediados de los años 90 comenzó refiriéndose principalmente a la venta de productos y servicios en Internet, básicamente mediante pago electrónico. La cantidad de comercio realizado por medios electrónicos ha crecido extraordinariamente desde la difusión de Internet [5]. Una gran variedad de comercio se realiza de esta manera, estimulando la creación y utilización de innovaciones como la transferencia electrónica de fondos, la gestión de la cadena de suministro, marketing en Internet, el procesamiento de transacciones en línea, intercambio electrónico de datos, sistemas de gestión de inventario y recopilación de datos automatizada.

El comercio electrónico (e-Commerce) también se define como la compra de productos de proveedores y su venta a clientes que utilizan las TIC. Entre los modelos de comercio electrónico más reconocidos tenemos empresa a empresa (B2B), empresa a consumidor (B2C) y empresas y gobierno (B2G). En un sentido amplio, negocios electrónicos (e-Business) cubre todo tipo de colaboraciones con socios comerciales, utilizando las TIC, y contempla los efectos legales. Una interacción de negocios es el intercambio electrónico de documentos comerciales o un mensaje que contiene un documento de negocios vital en un proceso empresarial; este tipo de actividades desempeña un papel clave en la colaboración. Desde finales de los años 1960, las empresas han utilizado los sistemas de información para el intercambio electrónico de datos con sus socios comerciales.

La integración electrónica ha dado lugar a cambios dramáticos en la definición de una empresa 
con el surgimiento de empresas virtuales cuyas capacidades para ofrecer sus productos al mercado se definen en gran medida por su capacidad para organizar y mantener una red de relaciones de negocios, en lugar de por su capacidad para fabricar un producto o prestar un servicio. Para entender una empresa individual, es necesario estudiar las redes de negocios en que está inmersa [6].

Existe un consenso sobre la influencia en Internet como plataforma de desarrollo de canales alternativos y/o complementarios de distribución. La proliferación del comercio electrónico puede estar relacionada con múltiples procesos de negocio [7]. Frente a la evidencia de que la Web resultó ser un canal efectivo para el comercio, se han sugerido diversos puntos de vista sobre la forma en que las empresas deben desarrollar una estrategia de comercio electrónico.

\subsection{La empresa como sistema y la integración de la arquitectura de soluciones de e-business}

Analizar a la empresa como sistema abierto ha sido propuesto por diversos autores, tomando como base los preceptos del biólogo Ludwig von Bertalanffy [8] al definir que el notable y a la vez improbable proceso de permanente equilibrio e incrementado nivel de organización de los sistemas vivos y de muchas de las estructuras sociales, económicas e industriales creadas por el hombre no podía ser explicado bajo la perspectiva de una entropía creciente. La razón de ello habría que buscarla en el hecho de que estos sistemas interaccionan con su entorno: son sistemas abiertos. Estos sistemas intercambian con su entorno flujos de materia, energía e información, y estos flujos marcan diferencias esenciales con los sistemas cerrados.

La empresa, vista como un sistema complejo, supera el enfoque analítico en el que se estudian de forma detallada y minuciosa las partes en un reducido ámbito de realidad, perdiéndose la visión de conjunto. Bajo el enfoque de sistemas se elaboran modelos que se utilizan en las decisiones empresariales para hacer manejables los sistemas, que permiten simplificar sus dimensiones operativas. La empresa está relacionada con su entorno, del cual recibe entradas en forma de recursos humanos, financieros, materiales, etc., que mediante la adecuada transformación permiten obtener unos resultados en forma de productos y/o servicios como salidas del sistema.

Las aplicaciones informáticas en la empresa han sido útiles en el logro de competitividad haciendo más eficiente su gestión; ejemplo de esto son las herramientas de ofimática, almacenamiento de datos, sistemas contables, sistemas de soporte de toma de decisiones, soluciones de comercio electrónico, Enterprise Resource Planning (ERP), redes sociales, etc.

En la figura 1 se concibe la arquitectura de una solución de comercio electrónico en el contexto de la WEB 2.0; esta debe tener en cuenta elementos de entornos como inversionistas, proveedores, competidores, canales de distribución y entidades de regulación que le permitan satisfacer al cliente externo, con una estructura organizacional coordinada (clientes internos) orientada a crear valor para clientes y accionista. La estructura empresarial está compuesta por los subsistemas de dirección y gestión, productivo, talento humano, financiero y comercial.

Esta arquitectura propuesta no puede alejarse del concepto de planificación de recursos empresariales (Enterprise Resource Planning - ERP) y de sus ventajas por tratarse de una propuesta integradora, flexible, escalable y que facilita la planeación de capacidades para la toma de decisiones [9]. Un elemento clave es la infraestructura tecnológica que debe ser gestionada con sus activos más importantes, la gente, la conectividad, el hardware, aplicaciones y portal web. Esta infraestructura debe estar orientada a una gestión de la relación con el cliente (Customer Relationship Management-CRM) [10] utilizando las potencialidades de Internet e-CRM. 


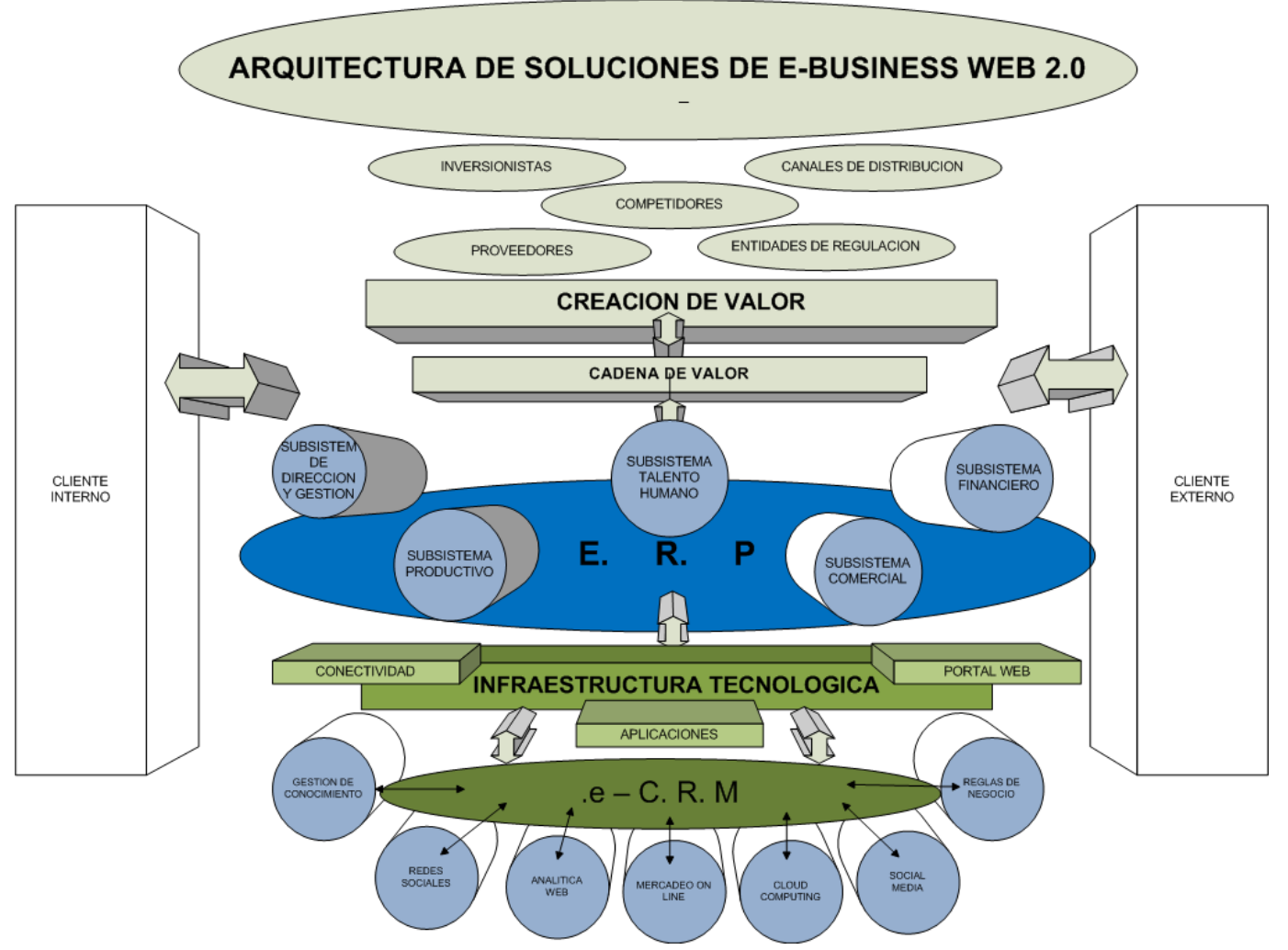

Figura 1. Arquitectura de una solución de Comercio Electrónico en el contexto de la WEB 2.0.

Fuente: elaboración propia.

También debe contemplar en su funcionalidad aspectos clave en el contexto de la Web 2.0: redes sociales en virtud de las posibilidades de interacción dinámica con clientes e interesados [11], analítica web como herramienta de toma de decisiones a partir de indicadores de accesibilidad en la Web [5], mercadeo en línea, computación en la nube como infraestructura tecnológica disponible con moderada inversión [12], social media, reglas y procesos de negocio y gestión de conocimiento [13].

\section{MODELO DE ORGANIZACIÓN PARA LA PLANEACIÓN ESTRATÉGICA DEL E-COMMERCE (MODELO PESCE)}

La herramienta se sustenta en los conceptos de planeación estratégica y mapas de conocimiento como instrumento para reducir la complejidad
[14], atendiendo los lineamientos relacionados con tecnología para un diagnóstico organizacional con el fin de determinar si la empresa es apta o no para implementar soluciones de comercio electrónico.

La integración de sistemas e infraestructuras de TIC en el proceso de gestión es esencial para obtener resultados efectivos, y refleja la visión estratégica de la organización. Sin embargo, la identificación de las necesidades de la organización está lejos de ocurrir en una manera directa; las políticas dentro de la organización y los intereses e intenciones de los participantes afectan la difusión e implantación de las innovaciones en las TIC [15]. En este sentido se propone un instrumento que viabilice una implementación favorable para la empresa y así garantizar el éxito de la implementación de soluciones de comercio electrónico. 
El procedimiento de evaluación de condiciones se desarrolla en tres etapas que se detallan en la figura 2:

- Formulación.

- Organización.

- Implementación y control.

\subsection{Formulación}

Inicia con el conocimiento de la empresa a través de la misión y la visión, seguido por la formulación de objetivos a corto mediano y largo plazo; se propone un análisis interno y externo de la

\section{Modelo PESCE}



Figura 2. Modelo PESCE - Planeación Estratégica del e-Commerce.

Fuente: elaboración propia. 
empresa. Con el enfoque del análisis del entorno competitivo se obtiene una evaluación y elección de estrategias orientadas a la implementación de soluciones de comercio electrónico. Se proponen preguntas orientadoras, como lo muestra la tabla 1, a la misión y la visión extrayendo puntos clave que indiquen si la organización en estudio es apta para la implementación de soluciones de comercio electrónico.

Los objetivos a corto, mediano y largo plazo se utilizan como instrumento para proponer las actividades de la empresa, estableciendo metas a corto, mediano y largo plazo. Los objetivos nos son estáticos, por lo tanto, se pueden modificar en relación con las necesidades de la empresa la cual debe revisarlos constantemente frente a los cambios que exige el entorno con respecto a la tecnología.

Una vez la empresa tenga establecidos la misión, la visión y los objetivos, debe hacer un análisis interno y externo con un enfoque competitivo de la organización, a su vez, realizar auditorías, internas y externas, y de esta manera llegar a la determinación de estrategias que conduzcan a la implementación del modelo de planeación estratégica de comercio electrónico.

El análisis externo es un aspecto clave de la planeación estratégica; factores exógenos como economía, política, tecnología, demografía, gobierno, cultura y sociedad son fuentes de oportunidades y amenazas; de allí la importancia de conocerlos y utilizarlos para obtener los objetivos empresariales. De la misma manera, el análisis interno pretende comprender las características que permitan cumplir los objetivos planteados, especificando debilidades y fortalezas e identificando cuáles son los recursos y capacidades tecnológicas de la organización. Finalmente en la etapa de formulación se hace una evaluación y una elección de las estrategias de acuerdo con los resultados obtenidos dentro del proceso de análisis de la organización.

\subsection{Organización}

Es la siguiente etapa a partir del resultado obtenido en la fase de formulación; los datos son empleados para la aplicación de la matriz de diagnóstico para implementación de soluciones de comercio electrónico; esta matriz proporciona coordenadas que luego serán ubicadas en un plano bidimensional y de acuerdo con la distribución de los puntos se podrá establecer si la empresa tiene o no condiciones para la implementación de una solución de comercio electrónico. Una vez evaluadas las condiciones de favorabilidad para una organización se toma como referente de planificación y estructuración de un proyecto tecnológico aplicando lo previsto en el "Diamante Organizacional y Tecnológico" propuesto.

Tabla 1. Preguntas orientadoras de la formulación.

\begin{tabular}{|c|l|l|}
\hline Qué & \multicolumn{1}{|c|}{ Cómo } & \multicolumn{1}{c|}{ Por qué } \\
\hline Misión & $\begin{array}{l}\text { La misión estará orientada hacia la empresa, proyectando la } \\
\text { singularidad de la organización. Identificando, seleccionan- } \\
\text { do e implantando las tecnologías que se acomoden mejor } \\
\text { al entorno y a los clientes, conservando una perspectiva } \\
\text { lucrativa. }\end{array}$ & $\begin{array}{l}\text { Para que la empresa se adapte al entorno, teniendo en } \\
\text { a través de implementación de soluciones de comercio } \\
\text { electrónico. }\end{array}$ \\
\hline Visión & $\begin{array}{l}\text { Analizar lo que la empresa busca crear y lograr a través de } \\
\text { la utilización de una Plataforma de comercio electrónico. }\end{array}$ & $\begin{array}{l}\text { Para darle un rumbo a la empresa, enfocándola hacia el } \\
\text { constante cambio e innovación en procura de la vanguardia } \\
\text { de la tecnología. }\end{array}$ \\
\hline
\end{tabular}

Fuente: elaboración propia. 


\subsubsection{Matriz de diagnóstico para implementación de soluciones de comercio electrónico}

Esta matriz es el resultado de una valoración cualitativa, a juicio de expertos, que valora el estado organizacional en cada uno de los factores críticos para la implementación de soluciones de comercio electrónico tales como arquitectura tecnológica, aplicaciones informáticas para Internet, personal capacitado y cultura tecnológica, como lo muestra la tabla 2. Se valora cualitativamente en un rango de cero (0) a cinco (5) el nivel de cumplimiento e integración con las variables provistas por la Planeación Estratégica (objetivos, análisis interno-externo, estrategias y entorno cliente-competencia); así, cero es el que indica una relación nula, y se incrementa sucesivamente hasta llegar a cinco como el valor más alto de relación entre las variables.

Después de calificar la empresa, estos valores son localizados en un plano bidimensional donde los factores críticos para la implementación de soluciones de comercio electrónico se ubican en el eje (x) y la valoración de expertos en el eje (y), de acuerdo con la figura 3 .

Los datos localizados en la figura 3 son ponderados y se establece la cantidad de valores que se localiza en cada uno de los rangos. Si el mayor porcentaje de los valores se localizan en el rango de cero (0) a dos (2) significa que la empresa no se encuentra preparada para la implementación de una solución de comercio electrónico; por lo tanto, podría hacer una revaluación de sus estrategias para volver a la etapa de formulación; si se localiza en el rango de cuatro a cinco se infiere que la empresa está posicionada en el mercado y seguramente ya tiene soluciones de este tipo; por último, si se localiza en el rango de dos a cuatro está en condiciones para la implementación de una solución de comercio electrónico.

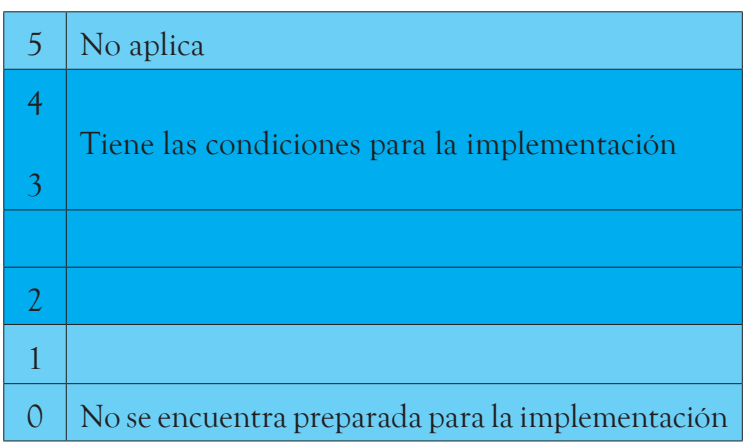

Figura 3. Rangos de aplicación organizacional de e-Commerce.

Fuente: elaboración propia.

\subsubsection{El diamante organizacional y tecnológico}

El diamante organizacional y tecnológico obedece en su diseño a la adaptación del diamante competitivo de Porter [16]; el mismo permite

Tabla 2. Matriz de diagnóstico para implementación de soluciones de comercio electrónico.

\begin{tabular}{|l|c|c|c|c|}
\hline \multicolumn{1}{|c|}{$\begin{array}{c}\text { Factores Críticos } \\
\text { Planeación Estratégica }\end{array}$} & $\begin{array}{c}\text { Arquitectura } \\
\text { Tecnológica } \\
\text { (A) }\end{array}$ & $\begin{array}{c}\text { Aplicaciones informáti- } \\
\text { cas para internet } \\
(B)\end{array}$ & $\begin{array}{c}\text { Personal } \\
\text { capacitado } \\
\text { (C) }\end{array}$ & $\begin{array}{c}\text { Cultura } \\
\text { tecnológica } \\
\text { (D) }\end{array}$ \\
\hline Objetivos & $0-5$ & $0-5$ & $0-5$ & $0-5$ \\
\hline Análisis Interno & $0-5$ & $0-5$ & $0-5$ & $0-5$ \\
\hline Análisis Externo & $0-5$ & $0-5$ & $0-5$ & $0-5$ \\
\hline Estrategias & $0-5$ & $0-5$ & $0-5$ & $0-5$ \\
\hline Entorno Cliente & $0-5$ & $0-5$ & $0-5$ & $0-5$ \\
\hline Entorno de Competencia & $0-5$ & $0-5$ & $0-5$ & $0-5$ \\
\hline
\end{tabular}

Fuente: elaboración propia. 
identificar las variables contingentes, las cuales se denominan como causales externas o de contexto de los cambios existentes en una organización; se trata de establecer la influencia de estas variables explicando la realidad organizacional y su nivel de influencia en la obtención de ventajas competitivas. Las mismas se definen a partir de la propuesta de Mintzberg [17] en lo referente a la estrategia y la cultura organizacional:

- La tecnología: se refiere a los recursos de los que disponemos, incluyendo técnicas, diseño, producción, procesos y tareas. Influye directamente en la base operativa de la empresa, la división de tareas, hardware, software, comunicación, humanware, redes, portales web, Internet, los cuales varían según el tipo de organización.

- El tamaño: afecta la estructura de la empresa, ya que la influencia de la dimensión en la empresa determina las unidades organizativas y la complejidad de la estructura.

- La antigüedad: la edad de la empresa influye en el diseño, funciones y la formalización de la organización. Esta variable conjuntamente con la de tamaño afectan en gran medida a la dirección intermedia y al staff directivo, lo cuales son esenciales para que se cumplan con efectividad, especialización y diferenciación los procesos en la organización.

- Poder: condiciona la actuación y el proceso de toma de decisiones según la estructura y jerarquías, y depende de donde se situé la variable, es decir, si es externa o interna.

- Propiedad: según el tipo de organización pública, privada o mixta determina la actuación y el diseño de la organización; tiende a afectar directamente a la alta dirección.

- Cultura: los valores, creencias, el estilo de liderazgo, las normas formales e informales, los procedimientos y las características generales de los miembros de la empresa condicionan el diseño y los resultados de la organización, establecen los procesos de funcionalidad y recogen los estilos de comunicación.

- Entorno: representa aquel que se adaptará o desaparecerá del mercado; esta variable nos explica la interacción de la empresa con el entorno.

Una organización que busque implementar soluciones de comercio electrónico debe controlar lo señalado en el Diamante Organizacional y Tecnológico: la cultura tecnológica, la estructura organizacional, la visión estratégica y las ventas y el marketing.

- Cultura tecnológica: la cual converge con el desarrollo tecnológico, a través de cambio, innovación, infraestructura, en concordancia con la clase de clientes que tiene la organización respecto a su planeación; todo esto conlleva a la repotenciación de la cultura tecnológica de la empresa con todas las variables la afectan, y permiten una reorganización que se adapte a la necesidades tecnológicas que el medio exige, para de esta manera, llegar a la implementación de soluciones de comercio electrónico. El factor crítico para implementar procesos innovadores sustentados en modelos de comercio electrónico es la apropiación de TIC por parte de la compañía y la capacitación del recurso humano en el manejo de esas herramientas [18].

- Estructura organizacional: con los factores que afectan a la implementación de soluciones de comercio electrónico se conduce a la organización a adoptar estrategias para el mejoramiento de su estructura a través de la división del trabajo, especialización de tareas, utilizando el recurso humano, sus habilidades y conocimientos, para llegar a la estandarización de procesos de trabajo; de esta manera se evidencian nuevos roles, funciones, cargos que permitan a la organización orientarse con seguridad a la tecnología propuesta. 
- Visión estratégica: propende por que la empresa se adapte al entorno competitivo y tecnológico; que sea una organización flexible, y que utilice la información como una cadena de conocimiento e interacción con el medio interno y externo; que garantice la toma correcta de las decisiones contempladas en la visión de la empresa y de acuerdo con una ideología que permita entender la organización como una realidad cambiante y dinámica que se enfoca hacia el futuro, acorde con la percepción del entorno.

- Venta y marketing: permite a la empresa que esta área, a través del rediseño de tareas, sea competitiva, interactiva, para que actúe en forma eficiente con el entorno, los proveedores, y clientes, en relación con la oferta y la demanda que establece el mercado, brindando soluciones eficientes, a tiempo y en línea a los clientes que lo requieran. Adicionalmente, se adaptan elementos convergencia digital, computación en la nube, redes sociales, etc. El plan de negocio en Internet es vital en esta etapa, a fin de priorizar los productos y/o servicios a ser comercializados por Internet, así como los beneficios percibidos por los clientes como, seguridad de las transacciones, privacidad de la información, y condiciones de equidad, una vez los consumidores en línea entran en fase de intercambio de información con el sitio Web [19].

El diamante organizacional y tecnológico (figura 4), permite una abstracción de los factores que afectan directamente la implementación de soluciones de comercio electrónico en la organización.

\subsubsection{Implementación y control}

Es la adopción de las estrategias que posibilitan la implementación de plataformas de comercio electrónico en cualquier tipo de organización; debe tener en cuenta aspectos como el enfoque de procesos, la adaptación al cambio y una visión flexible e innovadora. En esta etapa se deben atender criterios como:

- Capacitación: es necesario que las personas involucradas en el proceso cuenten con el conocimiento necesario para el manejo de la solución.

- Presupuesto de implementación: la empresa objeto de estudio en la implementación de soluciones de comercio electrónico previamente debe realizar una evaluación de los recursos existentes (inventario tecnológico) que permita especificar las inversiones y la evaluación costo beneficio de las mismas.

- Implementación de soluciones de comercio electrónico: previa evaluación de las condiciones existentes atendiendo la metodología propuesta se procede a adaptar las soluciones. Internet es un medio con dos aplicaciones: comunicación y transacción. Muchos autores consideran que la reducción de los costes asociados a estas dos funciones constituye el principal valor añadido de esta tecnología. Del mismo modo a lo que ocurre en los mercados de consumo, las empresas hacen uso de Internet en sus compras como una fuente de información $y$, a veces, como un canal de operación.

- Adecuación tecnológica al modelo de negocio: la planeación estratégica debe orientar este proceso en función de los recursos, capacidades internas, oportunidades y riesgos definidos en el análisis interno y externo, estableciendo técnicas en las que la adecuación tecnológica de la organización permita cumplir con los objetivos empresariales y soportar el modelo de negocio, definido este como una descripción de los roles y las relaciones entre los consumidores de una empresa, clientes, aliados y proveedores que identifican la información importante, flujos de producto y dinero, así como beneficios para los participante [20]. 


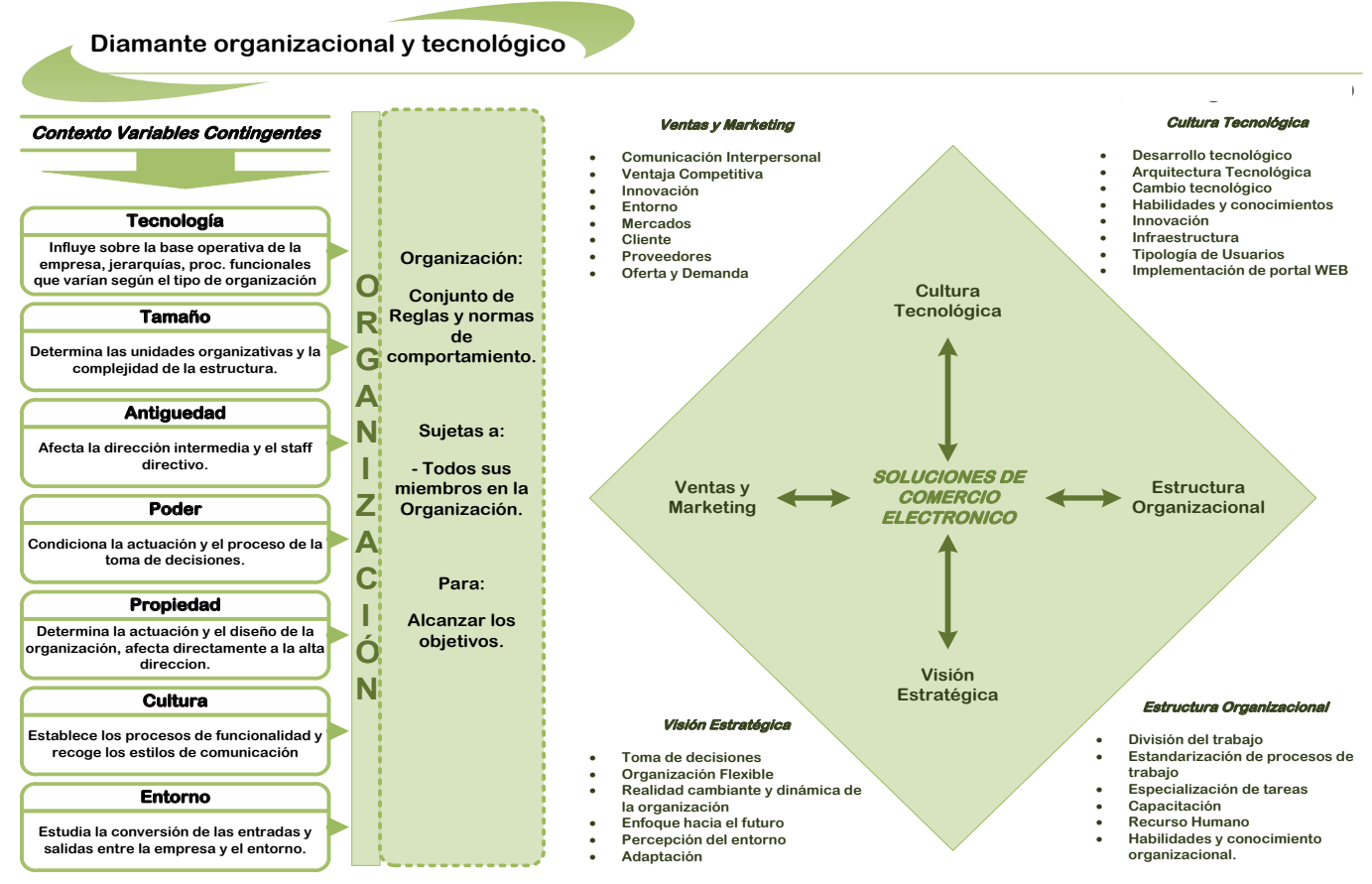

Figura 4. Diamante organizacional y tecnológico.

Fuente: elaboración propia

\section{VALIDACIÓN DEL MODELO}

Para validar el modelo PESCE, se realizó una encuesta para detectar la apropiación tecnológica en el sector textil de la ciudad de Bogotá, Colombia. El análisis de la cadena productiva se realizó a través de la aplicación de un formulario virtual a una muestra de 62 empresas pertenecientes al eslabón de transformación-confección del sector textil. Se encontró que solo el 37,1 \% de las empresas encuestadas usan Internet como vitrina para comercializar sus productos mediante un portal web, por lo cual se demuestra un desaprovechamiento de las tecnologías de la información teniendo en cuenta que todas afirmaron poseer como mínimo un computador personal (el 95,16 \% con acceso a Internet) y una cámara de fotografía digital (97 \%), por lo cual se detecta que existen herramientas para promocionar sus productos en la Web.

En cuanto a la realización de negocios en general se encontró que el 89 \% de los empresarios prefieren el pago en efectivo, el $68 \%$ prefiere el pago con tarjeta débito o crédito, y el $65 \%$, las transacciones bancarias. A pesar de que se detecta una preferencia por los medios tradicionales de hacer negocios, los encuestados manifiestan interés por el comercio electrónico. Las empresas que han realizado negocios a través de la web afirman que sus motivaciones para hacerlo, en un $79 \%$, son la disminución de costos, el ahorro de tiempo en un $85 \%$ y la agilidad del servicio en un $66 \%$, por lo cual se detecta una percepción positiva. En general un $53 \%$ cree que incurre en un ahorro de dinero con la salvedad de que la realización de negocio debe ser con alguien de confianza (el 69,3\% de los encuestados manifestaron que importa mucho y el $23 \%$ que importa un poco). Se vislumbra un interés de las compañías en la comercialización dado el $84 \%$ que afirmaron que les gustaría encontrar nuevos clientes en Internet y el $71 \%$ que consideran Internet un medio factible para comercializar sus productos.

Con los datos obtenidos, haciendo uso del juicio de expertos, se procesaron los datos en las 
matrices de diagnóstico y se encontró que del total de empresas el $8 \%$ está en el tercer rango de (4 a 5), con lo cual implementar una solución seguramente es un tema superado. El $71 \%$ tiene condiciones para la implementación y el $21 \%$ no cuenta con las condiciones para llevarla a cabo.

\section{CONCLUSIONES}

En general la comercialización de productos por medios electrónicos es una opción que no se debe descuidar por parte de las compañías encuestadas. El comercio electrónico es bien percibido y las mismas poseen la infraestructura que lo posibilita.Existen oportunidades de mejora en este sector.

La implementación de una metodología de planeación estratégica para organizaciones que orientan su acción transaccional en la Web garantizará la mejora sostenible en la estructuración e implantación de soluciones tecnológicas que fortalecen su modelo de negocio.

La metodología define una serie de parámetros a seguir para culminar con el conocimiento global de la empresa y, de esta manera, evaluarla, determinando las necesidades que esta tiene para la implementación de soluciones de comercio electrónico.

\section{REFERENCIAS BIBLIOGRAFÍCAS}

[1] Z. Qingfeng, C. Wenbo, and H. Lihua, "E-Business Transformation: An Analysis Framework Based on Critical Organizational Dimensions," Tsinghua Science and Technology, vol. 13, n. 3 , pp. 408-413, 2008.

[2] C. Llanos, B. Andres, and O. Angel, "An enterprise engineering approach for the alignment of business and information technology strategy," International Journal of Computer Integrated Manufacturing, vol. 24, n. ${ }^{\circ}$ 11, pp. 974-992, 2011.

[3] G. M. Tarazona -Bermudez, L. A. Rodriguez Rojas, and V. H. Medina, Modelos de Negocios Electronicos con Tecnologias WEB 2.0, 1 ${ }^{\text {ra }}$. Oviedo, 2012, p. 190.

[4] Grandon E. and Pearson M., "Electronic commerce adoption: an empirical study of small and medium US businesses," Information $\mathcal{E}$ Management, vol. 42, n. 1 , pp. 197-216, 2004.

[5] C. Carmona, S. Ramírez-Gallego, F. Torres, E. Bernal, M. J. del Jesus, and S. García, "Web usage mining to improve the design of an e-commerce website: OrOliveSur.com," Expert Systems with Applications, vol. 39, n. 12 , pp. 11243-11249, 2012.

[6] V. Zwass, "Electronic Commerce : Structures and Issues," International Journal of Electronic Commerce, vol. 1, n. ${ }^{\circ}$, pp. 3-23, 1996.

[7] F. F. Faroughian, S. P. Kalafatis, L. Ledden, P. Samouel, and M. H. Tsogas, "Value and risk in business-to-business e-banking," Industrial Marketing Management, vol. 41, n. 1 , pp. 68-81, Jan. 2012.

[8] B. Ludwing Von, Teoría general de los sistemas: fundamentos, desarrollo, aplicaciones, Segunda. Mexico, D.F.:, 2006, p. 356.

[9] A. Leon, Enterprise Resource Planning, Segunda Ed. New Delhi: Tata McGraw-Hill, 2008.

[10] G. Raab, R. Ajami, V. Gargeya, and G. J. Goddard, Customer Relationship Management: A global Perspective. Hampshire, England: Gower Publishing Limited,, 2008, p. 199.

[11] Y. Yu and K. Kong, "The research of enterprise boundary from the perspective of social network," E-Business and E-Government (ICEE), 2011 ..., 2011.

[12] S. Marston, Z. Li, S. Bandyopadhyay, J. Zhang, and A. Ghalsasi, "Cloud computing - The business perspective," Decision Support Systems, vol. 51, n. 1, pp. 176-189, Apr. 2011

[13] B. Öztayşi and T. Kaya, "Performance comparison based on customer relationship management using analytic network process," Expert Systems with Applications, vol. 38, n. ${ }^{\circ} 11$, pp. 9788-9798, 2011.

[14] I. Georgiou, "Messing about in transformations: Structured systemic planning for systemic solutions to systemic problems," European Journal of Operational Research, vol. 223, n. 2, pp. 392-406, Dec. 2012.

[15] A. Andrade and L. A. Joia, "Organizational structure and ICT strategies in the Brazilian Judiciary System," Government Information Quarterly, vol. 29, pp. S32-S42, Jan. 2012.

[16] M. E. Porter, On competition. Boston: 2008, p. 544.

[17] H. Mintzberg, The Strategy Process: Concepts, Con- 
texts, Cases: Global. Edinburgh Gate Harlow: , 2003, p. 489.

[18] G. M. Tarazona, L. Rodriguez, C. Pelayo-Garcia, and O. Sanjuan, "Model Innovation of Process Based on the Standard e-commerce International GS1," International Journal of Interactive Multimedia and Artificial Intelligence, vol. 1, n. ${ }^{\circ}$ 7, p. 70, 2012.
[19] H. Li, Han; Sarathy, Rathindra; Xu, "The role of affect and cognition on online consumers' decision to disclose personal information to unfamiliar online vendors," Decision Support Systems, vol. 51, n. ${ }^{\circ}$, pp. 434-445, 2011.

[20] M. A. Rashid, Z. Riaz, E. Sayin, H. A. Qureshi, G. Yilmaz, M. Shami, and H. Ping, "Strategic e-Commerce Model Driven-Architecture for e-Learning : TQM \& e-ERP Perspective," 2011. 